\title{
The role of combined systemic retinoids and phototherapy in the management of intermediate stage CTCL
}

\author{
M Fox, T Anderson
}

\begin{abstract}
Citation
$\mathrm{M}$ Fox, $\mathrm{T}$ Anderson. The role of combined systemic retinoids and phototherapy in the management of intermediate stage $C T C L$. The Internet Journal of Dermatology. 2008 Volume 7 Number 3.
\end{abstract}

DOI: $\underline{10.5580 / 99 \mathrm{~d}}$

\begin{abstract}
Dr. Anderson is an Associate Professor and Director of Photo Medicine for the Department of Dermatology at the University of Michigan Medical Center in Ann Arbor, Michigan. He is also the Medical Director of the Dermatology Satellite office, located at our new facilities at Domino's Farms. He is also the Medical Director of the U-M Dermatology Treatment Center of Ann Arbor and formerly the Chief of the Dermatology Service at the Ann Arbor Veteran's Adminstration Hospital from 1986 to 1993. He is currently Co-Director of the Multidisciplinary Cutaneous Lymphoma Program.
\end{abstract}

Dr Anderson is a member of the American Academy of Dermatology, American Dermatological Association, American Society For Photobiology, International Pigment Cell Society, International Society for Cutaneous Lymphoma, Photomedicine Society, Society For Investigative Dermatology, Medical Advisory Board for the Cutaneous Lymphoma Foundation, and past president of the Michigan Dermatological Society.

In recognition of his expertise in the diagnosis and treatment of skin diseases, Dr. Anderson has been listed by Best Doctors in America ${ }^{\circledR}$ since 1994.

\section{INTRODUCTION}

Cutaneous T-cell lymphoma (CTCL), the most common subtype of primary cutaneous lymphomas, is an umbrella term describing a heterogeneous group of distinct disease entities generally characterized by epidermotropic malignant T-lymphocytes. The variability in clinical and histologic features of CTCL is mirrored by the complexity and rapidly evolving treatment arsenal employed in managing this condition.

\section{INITIAL PRESENTATION}

To illustrate the role of adjunct systemic retinoids and phototherapy in the management of intermediate stage CTCL, we present the case of a 60 year old man who initially presented to the University of Michigan Department of Dermatology with a 6 month history of an asymptomatic, erythematous, minimally scaly eruption involving his abdomen, back and proximal upper and lower extremities.

\section{CONSIDERATIONS FOR DERM/ONC FROM REFERRING PHYSICIAN:}

Previous biopsies had shown, first, irregular acanthosis with focal spongiosis most consistent with subacute nummular dermatitis and, later, a chronic and lichenoid dermatitis suggestive of parapsoriasis. Prior to his presentation to our clinic, he had been treated with salicylic acid cream, hydrocortisone $2.5 \%$ cream, and over-the-counter emollients, none of which were noted to dramatically improve or halt the progression of his eruption. He was thus referred to a CTCL center for evaluation of refractory lesions thought to be consistent with MF.

At the time of his presentation, the patient had otherwise been feeling well, with no associated fever, chills, unintentional weight loss or night sweats. The remainder of his review of systems was negative. His past medical history was significant for hypertension, type 2 diabetes mellitus, dyslipidemia, gout and remote tobacco use. Daily medications included lisinopril, metformin, ezetimibesimvastatin, allopurinol and low-dose aspirin. He reported no family history of leukemia or lymphoma.

Total body skin and lymph node exam initially revealed mildly scaly erythematous patches and thin plaques on the 
abdomen, back and flanks, with extension to the proximal arms and upper thighs. Total body surface area involved was estimated to be 15-20 percent. No lymphadenopathy or hepatosplenomegaly was noted. Comprehensive metabolic profile, complete blood counts and LDH were generally within normal limits, with exception of a mildly elevated glucose.

\section{HISTOLOGY}

Biopsy was performed of a representative plaque on the left back and revealed a band-like atypical lymphoid infiltrate with epidermotropism. Immunohistochemical stains demonstrated CD3 and CD5-positive T cells with a CD4:CD8 ratio of 3-4:1, as well as reduced expression of CD7. T-cell receptor gamma gene rearrangement analysis failed to reveal clonality. Histologic and immunohistochemical features were consistent with mycosis fungoides, even despite negative gene rearrangement study results.

\section{MULTIDISCIPLINARY TEAM CONSIDERATIONS:}

Discussion of the patient's case at our multidisciplinary cutaneous lymphoma board, with input from dermatology, dermatopathology, medical oncology and radiation oncology, rendered a diagnosis of cutaneous T-cell lymphoma (mycosis fungoides), stage 1B (T2N0M0B0).

Consensus treatment recommendations from our multidisciplinary group included initiation of narrow band UVB phototherapy (NB-UVB) three times weekly until clinical remission was noted, followed by a slow taper, initially planned as twice weekly treatments for two months, then once weekly treatments for an additional 2 months, then once every other week treatments for one to two final months, as well as adjuvant triamcinolone $0.1 \%$ cream to be applied to affected areas in a burst and taper fashion.

\section{CLINICAL EVIDENCE AND MANAGEMENT ISSUES}

Narrow band UVB with adjuvant mid-potency topical steroid therapy was noted to produce excellent clinical response initially. However, upon eventually tapering to once weekly phototherapy, the patient's patches and thin plaques began to return. We returned to three times weekly NB-UVB treatments until clinical remission was again noted. However, again, upon tapering slowly to once weekly treatments per the aforementioned schedule, the patient again flared. After two flares even with extended phototherapy taper, we decided to add a systemic retinoid to his regimen as an adjuvant treatment modality. Acitretin was chosen given its relatively benign side effect profile and its support in the literature for management of other inflammatory T-cell mediated skin disease. With combined treatment of acitretin at dose of $25 \mathrm{mg}$ daily and three times weekly NB-UVB phototherapy, the patient again achieved clinical remission, this time in 6 weeks. He has since tapered to once weekly treatments without the return of clinically active CTCL. His liver and kidney function, blood counts and cholesterol panel are routinely monitored, so far without significant abnormality. He will continue to follow up in our clinic regularly.

\section{TEACHING POINTS AND TREATMENT GUIDELINES:}

Election of any particular treatment often hinges on a modality's ability to halt or delay disease progression in a way that minimizes side effects and toxicity. Options for early stage treatment for mycosis fungoides (MF), the most common variant of CTCL, include topical steroids, topical nitrogen mustard, total skin electron beam irradiation, phototherapy (UVB) and photochemotherapy (psoralen plus UVA [PUVA]).

Particularly in the management of more advanced or recalcitrant CTCL, an ever-evolving collection of systemic agents is often used. Oral retinoids are one such agent, and are major biologic response modifiers that can be combined with other therapies to improve response rates.

The therapeutic benefit of PUVA in managing MF is well known. Narrowband UVB (NB-UVB) therapy has also been shown to be a safe and efficacious treatment modality for early MF, with one study suggesting several advantages of NB-UVB therapy over treatment with broadband UVB and PUVA.1 The European Organization for Research and Treatment of Cancer (EORTC) guidelines for treatment of mycosis fungoides relate in particular that both narrow band UVB (NB-UVB) and PUVA are indicated in the management of early stage MF, NB-UVB for patch stage and PUVA for plaque stage. 2

Despite the well-documented efficacy of phototherapy in producing clinical remission, it is not infrequent for patients to slowly or incompletely respond to this treatment modality as monotherapy. In such cases, in our experience the combination of systemic retinoids and ultraviolet light is a safe and effective method of yielding improvement both in short term disease burden and in achieving long term clinical 
remissions. Our clinical experience is supported in the dermatologic literature.

Several studies regarding the management of psoriasis, another chronic (but benign) T-cell mediated skin disease, have demonstrated that when acitretin and phototherapy or photochemotherapy are combined, treatment efficacy is enhanced and treatment frequency, duration and cumulative doses are reduced when compared to either modality as monotherapy. 3 This synergistic effect has also been found to be beneficial in the management of CTCL. For example, the combination of low dose oral bexarotene and PUVA has been demonstrated to lead to an improved response in patients who had previously failed single-agent treatment regimens.4 One study demonstrated equal response rates with the combination of oral retinoids and PUVA versus PUVA alone, with longer remissions obtained with fewer PUVA sessions and lower UVA dosage if PUVA was combined with retinoids. 5

\section{CONCLUSIONS}

The management of this patient with a history of intermediate stage CTCL demonstrates the value of the combination of oral retinoids and phototherapy in achieving an expeditious and prolonged clinical remission when phototherapy as monotherapy had been unable to do so. Adding a low dose oral retinoid such as acitretin is a generally safe and effective next step in the treatment algorithm for patients with intermediate stage CTCL who prove to be slow to achieve clinical remission with phototherapy as monotherapy or for whom it is difficult to maintain prolonged clinical remission even with slowly tapered phototherapy. It has been our experience that UVB phototherapy in combination with systemic retinoids, either acitretin or bexarotene, (Re-UVB) works at least as well as PUVA in the management of early stage CTCL, and that retinoids in combination with PUVA (Re-PUVA) works better than PUVA alone in recalcitrant cases and often produces prolonged clinical remissions.

\section{References}

1. Diederen PV, van Weelden H, Sanders CJ, Toonstra J, van Vloten WA. Narrowband UVB and psoralen-UVA in the treatment of early-stage mycosis fungoides: a retrospective study. J Am Acad Dermatol. 2003 Feb;48(2):215-9

2. Trautinger F, Knobler R, Willemze R, Peris K, Stadler R, Laroche L, D'Incan M, Ranki A, Pimpinelli N, OrtizRomero P, Dummer R, Estrach T, Whittaker S. EORTC consensus recommendations for the treatment of mycosis fungoides/Sézary syndrome. Eur J Cancer. 2006 May;42(8):1014-30. Epub 2006 Mar 30.

3. Lebwohl M, Drake L, Menter A, Koo J, Gottlieb AB, Zanolli M, Young M, McClelland P. Consensus conference: acitretin in combination with UVB or PUVA in the treatment of psoriasis. J Am Acad Dermatol. 2001 Oct;45(4):544-53.

4. Singh F, Lebwohl MG. Cutaneous T-cell lymphoma treatment using bexarotene and PUVA: a case series. J Am Acad Dermatol. 2004 Oct;51(4):570-3.

5. Thomsen K, Hammar H, Molin L, Volden G. Retinoids plus PUVA (RePUVA) and PUVA in mycosis fungoides, plaque stage. A report from the Scandinavian Mycosis Fungoides Group. Acta Derm Venereol. 1989;69(6):536-8. 

CTCL

\section{Author Information}

Matthew C Fox, MD

Department of Dermatology, University of Michigan

Thomas F Anderson, MD

Department of Dermatology, University of Michigan 